\title{
Micromagnetic study of electrical-field-assisted magnetization switching in MTJ devices
}

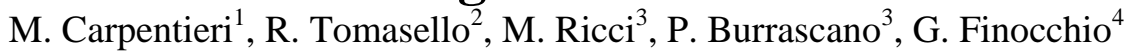

\author{
${ }^{1}$ Department of Electrical and Information Engineering, Politecnico of Bari, via E. Orabona 4, I-70125 Bari, Italy \\ ${ }^{2}$ Department of Computer Science, Modelling, Electronics and System Science, University of Calabria, Rende (CS), Italy \\ ${ }^{3}$ Department of Engineering, Polo Scientifico Didattico di Terni, University of Perugia, Terni, TR, I-50100 Italy \\ ${ }^{4}$ Department of Electronic Engineering, Industrial Chemistry and Engineering, University of Messina, Messina, Italy
}

\begin{abstract}
Perpendicular MgO-based Magnetic Tunnel Junctions are optimal candidates as building block of Spin Transfer Torque (STT) magnetoresistive memories. However, up to now, the only STT is not enough to achieve switching current density below $10^{6} \mathrm{~A} / \mathrm{cm}^{2}$. A recent work [Wang et al., Nature Mater., vol. 11, pp 64-68, Jan. 2012] has experimentally demonstrated the possibility to perform magnetization switching assisted by an electric-field at ultra-low current density. Theoretically, this switching has been studied by using a macrospin approach only. Here, we show a full micromagnetic study. We found that the switching occurs via a complex nucleation process including the nucleation of magnetic vortexes.
\end{abstract}

Index Terms-Magnetization switching, interfacial perpendicular anisotropy, voltage controlled magnetocrystalline anisotropy.

\section{INTRODUCTION}

$\mathrm{M}$ AGNETIC Tunnel Junctions

(MTJs) with a ferromagnetic $\mathrm{CoFeB}$ free layer and an $\mathrm{MgO}$ spacer, have excellent characteristics to be used in Spin Transfer Torque Magnetoresistive Random Access Memories (STTMRAM) [1], [2]. The magnetization reversal induced by spin torque was firstly observed in in-plane structures [3], [4] and, therefore, it was successfully achieved in perpendicular devices [5], [6]. Reductions of the critical current and high thermal stability have been obtained with different strategies [7], [8], [9], [10], [11], [12] on top of those the use of interfacial perpendicular anisotropy arising at the interface between Fe-rich $\mathrm{CoFeB}$ and $\mathrm{MgO}$ [13], [14], [15]. However, the reversal current density is still too high, hence, besides the only STT, other switching mechanisms have to be investigated. Many experimental [16], [17], and macrospin [18] studies have addressed the magnetization reversal assisted by the voltage controlled magnetocrystalline anisotropy (VCMA) [19] and, among them, a milestone experiment was performed by Wang et al. [20]. They have demonstrated that switching processes can be controlled by a trade-off between a bias field $H_{e x t}$ and an unipolar voltage pulse, achieving the magnetization switching for current densities of the order of $10^{4} \mathrm{~A} / \mathrm{cm}^{2}$ in presence of a small bias field $H_{\text {ext }}(+\mathrm{Z}$ direction)For the initial parallel state (both magnetization along -z direction), a low voltage pulse is applied to decreases the perpendicular anisotropy in order that the current flowing through the stack switch the state from the parallel (P) to antiparallel (AP). The $\mathrm{P} \rightarrow \mathrm{AP}$ switching is assisted by the external field. For AP-->P switching the scenario is different. A high voltage pulse reduces drastically the perpendicular anisotropy, leading to an in-plane free layer magnetization. In this case, the STT related to the large current density flowing through the MTJ stack switch the magnetization. Here, we perform a numerical study by means of micromagnetic simulations to deeply understand that experiment[20]. Our results predict the achievement of electrical-assistedmagnetization-reversal in the nanosecond scale at $J=10^{5} \mathrm{~A} / \mathrm{cm}^{2}$, making such switching mechanism more suitable for high speed storage applications. The switching occurs via the nucleation of complex magnetization patterns, including vortex and antivortex and, therefore, it is strongly spatially non-uniform, making impossible its understanding in the framework of macrospin approximation.

The paper is organized as follows. Section II introduces the device and the numerical details. Section III describes the achieved results and explains the physical origin of the switching process. Conclusions are reported in Section IV.

\section{NUMERICAL MODEL}

We study an MTJ stack composed by $\mathrm{CoFeB}(1.3 \mathrm{~nm}) \backslash \mathrm{MgO}(1.2 \mathrm{~nm}) \backslash \mathrm{CoFeB}(1.6 \mathrm{~nm})$. The thinner and the thicker layers act as pinned and free layer respectively. A sketch of the structure is represented in Fig. 1. We introduce a Cartesian coordinate system with the $z$-axis oriented along the out-of-plane direction and the $x$ - and $y$-axis positioned into the film plane. The device has a circular cross section with a diameter $D=200 \mathrm{~nm}$, a free layer saturation magnetization $M_{s}=9 \times 10^{5} \mathrm{~A} / \mathrm{m}$ and an exchange constant $A=2 \times 10^{-11} \mathrm{~J} / \mathrm{m}$. Since the free layer is very thin and it is coupled to a $\mathrm{MgO}$ insulator, we can consider a VCMA $K_{u}=5.3 \times 10^{5} \mathrm{~J} / \mathrm{m}^{3}$ [13], which is large enough to stabilize an out-of-plane magnetization easy axis. The magnetization of the pinned layer points down (negative $z$-direction) and an external magnetic field $H_{e x t}=5.5 \mathrm{mT}$ is applied along the positive $z$ direction. Initially, the free and pinned layer magnetizations are collinear.

The voltage dependence of the anisotropy constant is linear as shown in [20]. The anisotropy constant is reduced to $K_{u}$ $=4.8 \times 10^{5} \mathrm{~J} / \mathrm{m}^{3}$ for the current pulse to switch $\mathrm{P} \rightarrow \mathrm{AP}$ while its value is to $4.4 \times 10^{5} \mathrm{~J} / \mathrm{m}^{3}$ for the current pulse to switch $\mathrm{AP} \rightarrow \mathrm{P}$.

\section{FIG. 1 HERE}

The micromagnetic simulations are based on the numerical solution of the Landau-Lifshitz-Gilbert-Slonczewski equation: 


$$
\begin{aligned}
& \left(1+\alpha^{2}\right) \frac{d \mathbf{m}}{d t}=-\left(\mathbf{m} \times \mathbf{h}_{\text {eff }}\right)-\alpha \mathbf{m} \times\left(\mathbf{m} \times \mathbf{h}_{\text {eff }}\right) \\
& -\frac{g\left|\mu_{\mathrm{B}}\right| J_{M T J}}{e \gamma_{0} M_{\mathrm{S}}^{2} d_{\mathrm{FL}}} \varepsilon\left(\mathbf{m}, \mathbf{m}_{p}\right)\left[\mathbf{m} \times\left(\mathbf{m} \times \mathbf{m}_{\mathrm{P}}\right)-q(V)\left(\mathbf{m} \times \mathbf{m}_{\mathrm{P}}\right)\right]
\end{aligned}
$$

where $\alpha$ is the Gilbert damping, $\mathbf{m}$ is the magnetization vector, $\mathbf{h}_{\text {eff }}$ is the effective field, $g$ is the Landè factor, $\mu_{\mathrm{B}}$ is the Bohr magneton, $e$ is the electron charge, $\gamma_{0}$ is the gyromagnetic ratio, $M_{\mathrm{S}}$ is the free layer saturation magnetization, $d_{\mathrm{FL}}$ is the thickness of the free layer, $J_{M T J}$ is the current density flowing perpendicular to the structure, $\varepsilon\left(\mathbf{m}, \mathbf{m}_{p}\right)$ characterizes the angular dependence of the Slonczewski spin torque term and $\mathbf{m}_{\mathrm{P}}$ is the magnetization of the pinned layer. $q(V)$ is the voltage dependent parameter for the perpendicular torque, where $V$ is the voltage computed from the bias voltage dependence of the TMR and the current density. We set $\alpha=0.015$, a typical value for this material [13]. The thermal field $\mathbf{h}_{t h}$, which is included as an additive term to the effective field, is a random fluctuating three-dimensional vector quantity, given by $\mathbf{h}_{t h}=\left(\xi / M_{s}\right) \sqrt{2\left(\alpha K_{B} T / \mu_{0} \gamma_{0} \Delta V M_{s} \Delta t\right)}$, where $K_{B}$ is the Boltzmann constant, $\Delta V$ is the volume of the computational cubic cell, $\Delta t$ is the simulation time step, $T$ is the temperature of the sample [21], and $\xi$ is a random number from a Gaussian distribution with zero mean and unit variance [22]. Detailed numerical description of the model and the algorithms can be found in [23], [24].

\section{RESULTS AND DISCUSSION}

Fig. 2a shows the current pulses (as consequence of voltage pulses) as function of time $t$. We consider that the switching phenomenon is achieved when the normalized $z$-component of the magnetization reaches \pm 0.8 . The voltage pulses give rise to an anisotropy constant reduction and a current flow through the MTJ. The first pulse, long $15 \mathrm{~ns}$, gives rise to a negative current density of about $6 \times 10^{5} \mathrm{~A} / \mathrm{cm}^{2}$. During this time range, the $z$-component of the magnetization starts to reverse (see Fig. 2b), remaining in an oscillatory state. At $t=18 \mathrm{~ns}$, when the first current pulse is switched off, $K_{u}$ is turned back to the initial value, and due to the external field $H_{\text {ext }}$ (pointing upwards), the $\mathrm{P} \rightarrow \mathrm{AP}$ switching is fully attained. This is a stable state and the $\left\langle m_{z}\right\rangle$ keeps firmly the AP state. At $t=38 \mathrm{~ns}$ the second higher pulse is applied $\left(J_{M T J}=-7 \times 10^{5} \mathrm{~A} / \mathrm{cm}^{2}\right)$ for a time of $3 \mathrm{~ns}$. Consequently, since $K_{u}$ reduces more and the negative current favors the $\mathrm{P}$ state, the AP state becomes unstable, accordingly $\left\langle m_{z}\right\rangle$ starts to switch back. When the current pulse is switched off ( $t=41 \mathrm{~ns})$, the anisotropy field returns to the initial value and helps the full magnetization reversal. At $t=47 \mathrm{~ns}$, the $\mathrm{AP} \rightarrow \mathrm{P}$ switching is achieved. The current densities to achieve both switching processes in less than $50 \mathrm{~ns}$ are about one order of magnitude smaller than the one used in STT-MRAM [14].

\section{FIG. 2 HERE}

The $\mathrm{P} \rightarrow \mathrm{AP}$ is approximately insensitive to the pulse time duration. With this in mind, we systematically study the $\mathrm{AP} \rightarrow \mathrm{P}$ switching as function of the duration of the current pulse. In detail, we sweep the pulse duration from 2.6 to $7 \mathrm{~ns}$, with a time step of $0.2 \mathrm{~ns}$, finding that only when the pulse time is 2.8, 3 and $5.6 \mathrm{~ns}$ the switching is obtained. In Fig. 3, we illustrate the temporal evolution of the three components $\left\langle m_{x}\right\rangle,\left\langle m_{y}\right\rangle$ and $\left\langle m_{z}\right\rangle$ of the magnetization when the pulse duration is $3 \mathrm{~ns}$ (Fig. 3a) and when it is $2.6 \mathrm{~ns}$ (Fig. 3b). After the current pulse is switched off, in the former case $\left\langle m_{z}\right\rangle$ switches from AP to $\mathrm{P}$, whereas in the latter one the reverse does not occur and $\left\langle m_{z}\right\rangle$ returns to the AP state $\left(\left\langle m_{z}\right\rangle=+1\right)$.

\section{FIG. 3 HERE}

The origin of such dependence can be understood by studying the spatial configuration of the magnetization before and after the current pulse is removed. Fig. 4 represents the spatial distribution of the magnetization in the case where the reversal occurs (Fig. 4a and $4 \mathrm{~b}$ ) and when it is not achieved (Fig. 4c and 4d). In out-of-plane MgO-based MTJs, the easyaxis of the magnetization is mainly dependent on the competition between the demagnetizing field $\mathbf{h}_{\mathrm{d}}$ and the perpendicular anisotropy field $\mathbf{h}_{K \perp}$. Particularly, the $z$ component of $\mathbf{h}_{\mathrm{d}}$ tries to lead the magnetization into the free layer plane, whereas $\mathbf{h}_{K \perp}$ keeps the magnetization in the outof-plane direction. Once we reduce the perpendicular anisotropy by applying the current pulse, $\mathbf{h}_{\mathrm{d}}$ becomes comparable with $\mathbf{h}_{K \perp}$, and, therefore, we obtain a quasi-inplane structure. In this framework, the applied current is able to excite non-uniform chiral magnetization configurations such as vortexes and antivortexes [25].

\section{FIG. 4 HERE}

When the current pulse is applied, the circular symmetry of the Oersted field nucleates a vortex state. In this case, two vortexes are generated and they rotate in the plane of the structure. Particularly, in Fig. $4 \mathrm{a}$ and $4 \mathrm{~b}$ we can clearly identify two vortexes and two antivortexes (indicated with "V" and "AV" respectively in the figures); on the other hand, in Fig. 4c and 4d, the second antivortex is pushed out from the free layer and only one antivortex is evident together with two vortexes. In addition, in the cases when the switching is obtained, a larger portion of the magnetization points down along the negative $z$-axis (Fig. $4 \mathrm{a}$ and $4 \mathrm{~b}$ show blue background color), with respect to the other two cases (Fig. 4c and 4d). After removing the current pulse, the spatial distribution of the magnetization evolves in a central domain oriented in the positive out-of-plane direction and in two side domains pointing along $-z$-direction (see Fig. $4 \mathrm{e}$ ). At a later stage, the two side domains collapse each other forming only one domain. Finally, it expands across the whole section of the free layer. A magnetization configuration similar to the previous case is also observed when the current pulse is long $2.6 \mathrm{~ns}$ (Fig. 4f), with the exception that the central domain is 
larger and the lateral domains smaller. Subsequently, the central domain expands, bringing back the magnetization in the AP state. After removing the current pulse, the vortex state (with $+\mathrm{z}$ polarity) is destroyed relaxing into a more uniform magnetization configuration, pointing upwards. The width of this region depends on the vortexes spatial position before removing the pulse. If the area of the nucleated central domain is confined in the centre of the free layer, the side domains (pointing downwards) are quite large to favour energetically the $-\mathrm{z}$ magnetization direction (case of Fig. 4e).

Just to summarize, the $\mathrm{AP} \rightarrow \mathrm{P}$ switching is achieved only in the case where two vortexes and two antivortexes are simultaneously nucleated just before the current pulse is switched off. On the other hand, when two vortexes and only one antivortex are generated, the $\mathrm{AP} \rightarrow \mathrm{P}$ switching does not occur. The number of chiral configurations (four or three) excited when the current pulse is removed depends on the pulse duration.

Finally, we introduce the thermal field as computed at room temperature $T=300 \mathrm{~K}$. Figure 5 shows the $\left\langle m_{z}\right\rangle$ behavior for different current pulse durations in the $\mathrm{P} \rightarrow \mathrm{AP}$ switching (Fig. 5a) and in the $\mathrm{AP} \rightarrow \mathrm{P}$ one (Fig. 5b). In the first reversal process, pulse durations from $6 \mathrm{~ns}$ to $15 \mathrm{~ns}$ are taken into account and in all the cases the magnetization is fully reversed within $30 \mathrm{~ns}$. On the other hand, the thermal effects make the second switching harder. In fact, only when the pulse durations are 3 and $6.2 \mathrm{~ns}$ the magnetization switching is obtained and for time larger than $80 \mathrm{~ns}$. Therefore, the thermal effect triggers the $\mathrm{P} \rightarrow \mathrm{AP}$ switching and delays the process for the $\mathrm{AP} \rightarrow \mathrm{P}$ one. This is related to the fact that the $\mathrm{P} \rightarrow \mathrm{AP}$ process is strongly dependent on the external field, while the $\mathrm{AP} \rightarrow \mathrm{P}$ is mainly related to STT.

\section{CONCLUSION}

In summary, the switching assisted by an electric field, studied experimentally in [20], has been described within a micromagnetic framework. The dependence of the entire switching process on the current pulse time duration has been analyzed, highlighting that the $\mathrm{AP} \rightarrow \mathrm{P}$ one occurs only when two vortexes and two antivortexes are simultaneously excited before the current pulse is switched off. Thus, the switching behavior is characterized by strongly non-uniform magnetization configurations and, for this reason, it is not possible to describe it by using a simple macrospin model. Furthermore, with respect to the experimental case, it has been shown how the whole magnetization reversal process can be executed in a time of about $50 \mathrm{~ns}$, and, by considering also the thermal effect at room temperature, within about $100 \mathrm{~ns}$ (much less than the experimental evidence). In this perspective, the results suggest that the electrical-field-assisted switching mechanism can be very useful and achievable from a technological point of view.

\section{ACKNOWLEDGMENT}

This work was supported by the project PRIN2010ECA8P3 from Italian MIUR. MR and PB acknowledge financial support from Fondazione CARIT. MC acknowledges financial support by the project "RES NOVAE - Reti, Edifici, Strade Nuovi Obiettivi Virtuosi per l'Ambiente e l'Energia". This project is supported by the Italian University and Research National Ministry research and competitiveness program that Italy is developing to promote "Smart Cities Communities and Social Innovation".

\section{REFERENCES}

[1] J. Åkerman, "Toward a Universal Memory," Science, vol. 308, p. 508, 2005.

[2] H. Li, X. Wang, Z.-L. Ong, W.-F. Wong, Y. Zhang, P. Wang, and Y. Chen, "Performance, power and reliability tradeoffs of STT-RAM cell subject to architecture-level requirement," IEEE Trans. Magn., vol. 47, p. 2356, October 2011.

[3] J. A. Katine, F. J. Albert, R. A. Buhrman, E. B. Myers and D. C. Ralph, "Current-driven magnetization reversal and spin-wave excitations in Co/Cu/Co pillars," Phys. Rev. Lett., vol. 84, pp. 3149-3152, Apr. 2000.

[4] Y.-T. Cui, G. Finocchio, C. Wang, J. A. Katine, R. A. Buhrman, and D. C. Ralph, "Single-Shot Time-Domain Studies of Spin-Torque-Driven Switching in Magnetic Tunnel Junctions," Phys. Rev. Lett., vol. 104, 097201, March 2010.

[5] S. Mangin, D. Ravelosona, J. A. Katine, M. J. Carey, B. D. Terris, E. E. Fullerton, "Current-induced magnetization reversal in nanopillars with perpendicular anisotropy," Nature Mater., vol. 5, pp. 210-215, March 2006.

[6] M. Carpentieri, G. Finocchio, B. Azzerboni, and L. Torres, "Spin transfertorque resonant switching and injection locking in the presence of a weak external microwave field for spin valves with perpendicular materials," Phys. Rev. B, vol. 82, 094434, September 2010.

[7] O. Ozatay, N. C. Emley, P. M. Braganca, A. G. F. Garcia, G. D. Fuchs, I. N. Krivorotov, R. A. Buhrman, and D. C. Ralph, "Spin transfer by nonuniform current injection into a nanomagnet," Appl. Phys. Lett., vol. 88, 202502, May 2005.

[8] P.M. Braganca, O. Ozatay, A. G. F. Garcia, O. J. Lee, D. C. Ralph, and R. A. Buhrman, "Enhancement in spin-torque efficiency by nonuniform spin current generated within a tapered nanopillar spin valve," Phys. Rev. B, vol. 77, 144423, Apr. 2008.

[9] Y.-T. Cui, J. C. Sankey, C. Wang, K. V. Thadani, Z.-P. Li, R. A. Buhrman, and D. C. Ralph, "Resonant spin-transfer-driven switching of magnetic devices assisted by microwave current pulses," Phys. Rev. B, vol. 77, 214440, June 2008.

[10] N. Reckers, J. Cucchiara, O. Posth, C. Hassel, F. M. Romer, R. Narkowicz, R. A. Gallardo, P. Landeros, H. Zahres, S. Mangin, J. A. Katine, E. E. Fullerton, G. Dumpich, R. Meckenstock, J. Lindner, and M. Farle, "Effect of microwave irradiation on spin-torque-driven magnetization precession in nanopillars with magnetic perpendicular anisotropy," Phys. Rev B, vol. 83, 184427, May 2011.

[11] M. Carpentieri, M. Ricci, P. Burrascano, L. Torres, and G. Finocchio, "Noise-like sequences to resonant excite the writing of a universal memory based on spin-transfer-torque MRAM," IEEE Trans. Magn., vol. 48, p. 2407, September 2012.

[12] M. Carpentieri, M. Ricci, P. Burrascano, L. Torres, and G. Finocchio, "Wideband microwave signal to trigger fast switching processes in magnetic tunnel junctions," J. Appl. Phys., vol. 111, p. 07C909, 2012.

[13] S. Ikeda, K. Miura, H. Yamamoto, K. Mizunuma, H. D. Gan, M. Endo, S. Kanai, J. Hayakawa, F. Matsukura, and H. Ohno, "A perpendicularanisotropy $\mathrm{CoFeB}-\mathrm{MgO}$ magnetic tunnel junction," Nature Mater., vol. 9, pp. 721-724, July 2010.

[14] P. Khalili Amiri, Z. M. Zeng, J. Langer, H. Zhao, G. Rowlands, Y.-J. Chen, I. N. Krivorotov, J.-P. Wang, H. W. Jiang, J. A. Katine, Y. Huai, K. Galatsis, and K. L. Wang, "Switching current reduction using perpendicular anisotropy in $\mathrm{CoFeB}-\mathrm{MgO}$ magnetic tunnel junctions," Appl. Phys. Lett., vol. 98, 112507, March 2011.

[15] R. Tomasello, V. Puliafito, B. Azzerboni, and G. Finocchio, "Switching properties in magnetic tunnel junctions with interfacial perpendicular anisotropy: micromagnetic study," http://arxiv.org/ftp/arxiv/papers/1402/1402.4352.pdf.

[16] S. Kanai, M. Yamanouchi, S. Ikeda, Y. Nakatani, F. Matsukura, and H. Ohno, "Electric field-induced magnetization reversal in a perpendicular- 
anisotropy $\mathrm{CoFeB}-\mathrm{MgO}$ magnetic tunnel junction," Appl. Phys. Lett., vol. 101, 122403, September 2012.

[17] S. Kanai , M. Yamanouchi, S. Ikeda, Y. Nakatani, F. Matsukura, and H. Ohno, "Electric Field-Induced Magnetization Switching in CoFeB$\mathrm{MgO}$ - Static Magnetic Field Angle Dependence," IEEE Trans. Magn., vol. 50, 4200103, January 2014.

[18] P. K. Amiri, P. Upadhyaya, J. G. Alzate, and K. L. Wang, "Electric-fieldinduced thermally assisted switching of monodomain magnetic bits," $J$. Appl. Phys., vol. 113, 013912, January 2013.

[19] T. Maruyama, Y. Shiota, T. Nozaki, K. Ohta, N. Toda, M. Mizuguchi, A. A. Tulapurkar, T. Shinjo, M. Shiraishi, S. Mizukami, Y. Ando, and Y. Suzuki, "Large voltage-induced magnetic anisotropy change in a few atomic layers of iron", Nature Nanotech., vol. 4, pp. 158-161, January 2009.

[20] W.G. Wang, M. Li, S. Hageman, and C. L. Chien, "Electric-fieldassisted switching in magnetic tunnel junctions", Nature Mater., vol. 11, pp. 64-68, January 2012.

[21] I.N. Krivorotov, N.C. Emley, A.G.F. Garcia, J.C. Sankey et al., "Temperature Dependence of Spin-Transfer-Induced Switching of Nanomagnets," Phys. Rev. Lett., vol. 93, 166603, 2004.

[22] W. F. Brown, "Thermal Fluctuations of a Single-Domain Particle,", Phys. Rev., vol. 130, p. 1677, 1963;

[23] A. Giordano, G. Finocchio, L. Torres, M. Carpentieri, and B. Azzerboni, "Semi-implicit integration scheme for Landau-Lifshitz-GilbertSlonczewski equation," J. Appl. Phys., vol. 111, 07D112, February 2012.

[24]L. Lopez-Diaz, D. Aurelio, L. Torres, E. Martinez, M.A. Hernandez Lopez, J. Gomez, O. Alejos, M. Carpentieri, G. Finocchio, and G. Consolo, "Micromagnetic simulations using Graphics Processing Units," J. Phys. D: Appl. Phys., vol. 45, 323001, July 2012.

[25] G. Finocchio, O. Ozatay, L. Torres, R. A. Buhrman, D. C. Ralph, and B. Azzerboni, "Spin-torque-induced rotational dynamics of a magnetic vortex dipole", Phys. Rev. B, vol. 78, 174408, November 2008. 


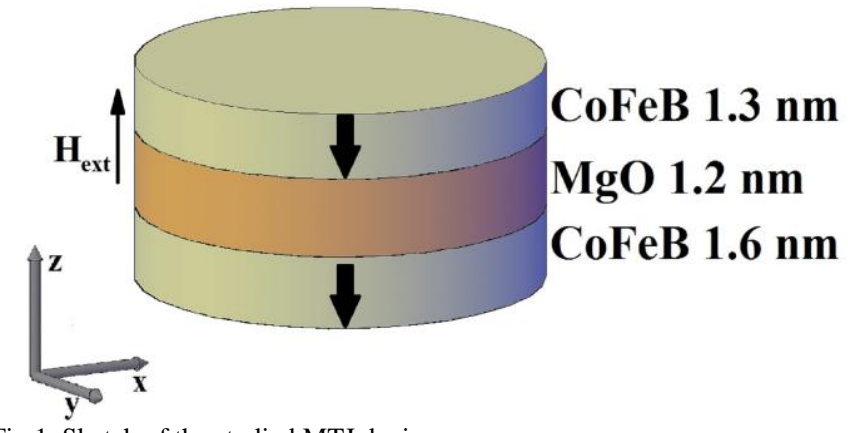

Fig.1. Sketch of the studied MTJ device.
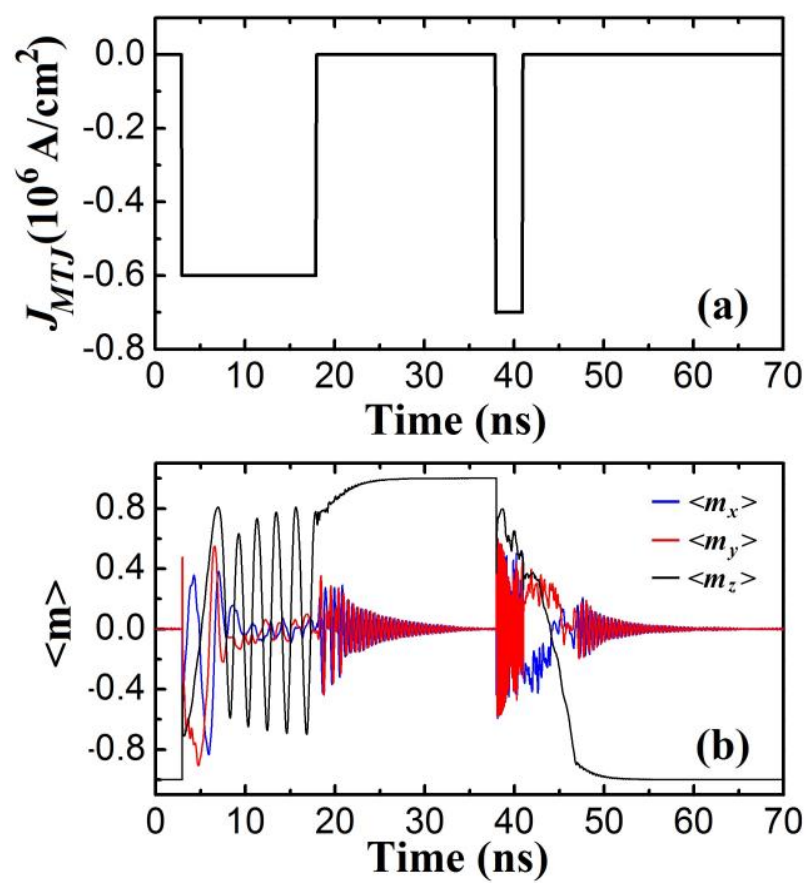

Fig. 2. (a) Applied current pulses. (b) Time domain plot of the three normalized components of the magnetization $\left(\left\langle m_{x}\right\rangle,\left\langle m_{y}\right\rangle\right.$ and $\left\langle m_{z}\right\rangle$, respectively represented in blue, red and black) during a whole reversal process ( $\mathrm{P}$ [ $\mathrm{AP}$ and $\mathrm{AP} ? \mathrm{P}$ ) as a function of the current pulses in (a).
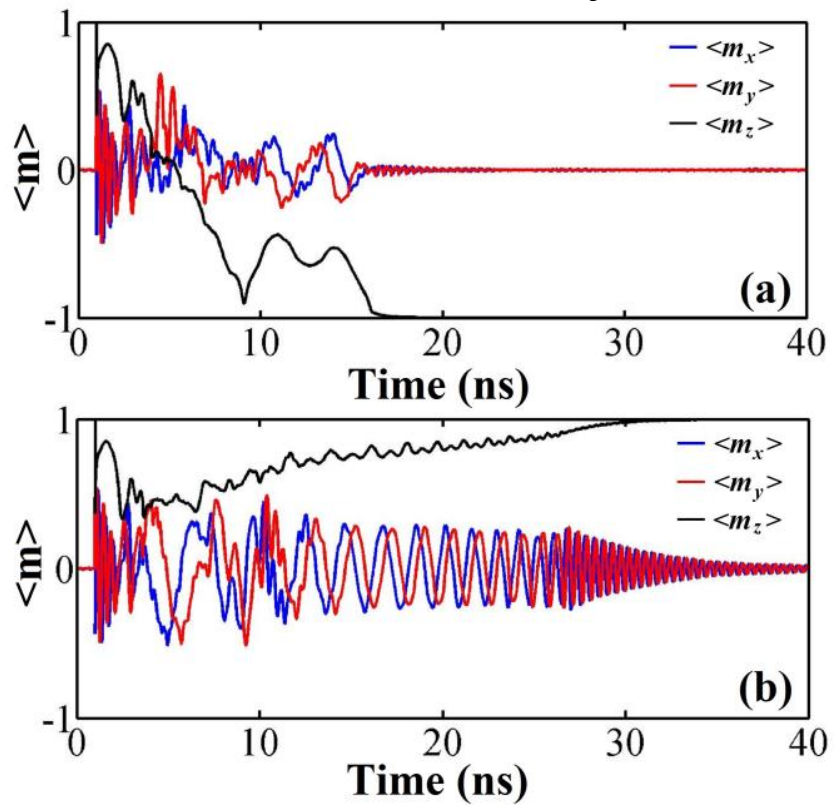

Fig. 3. Time domain plot for the three normalized components of the magnetization $\left\langle m_{x}\right\rangle,\left\langle m_{y}\right\rangle$ and $\left\langle m_{z}\right\rangle$, respectively represented in blue, red and black. (a) Pulse time of 3 ns. (b) Pulse time of 2.6 ns.

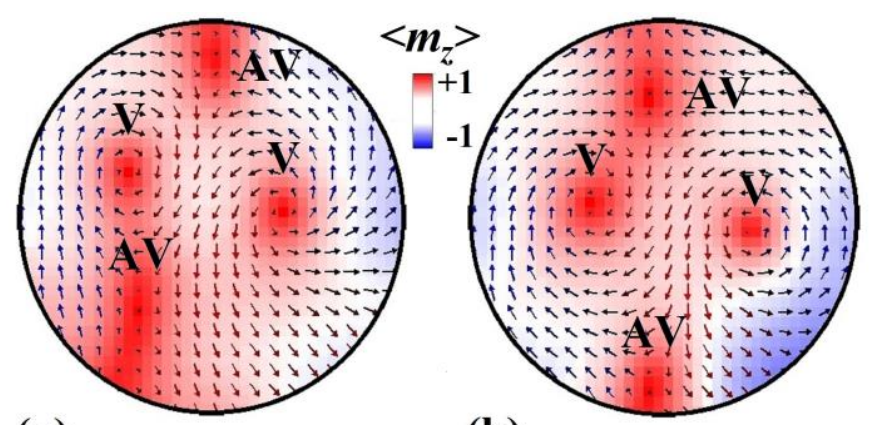

(a)

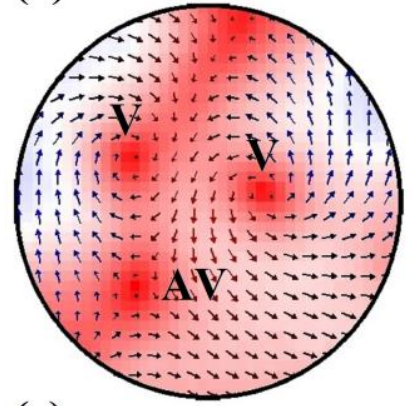

(c)

(b)

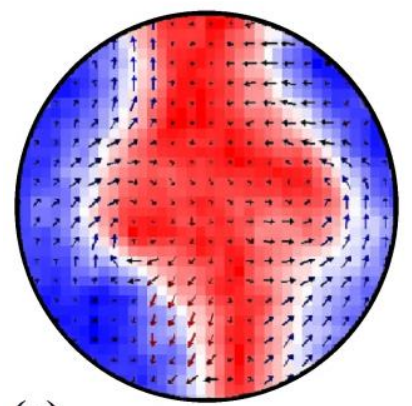

(e)

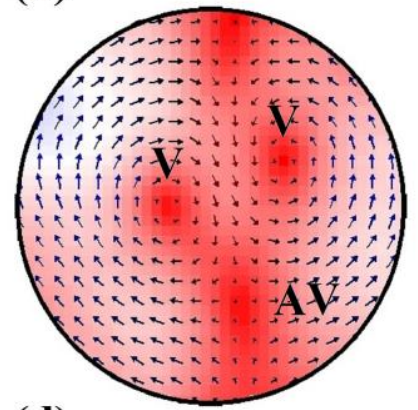

(d)

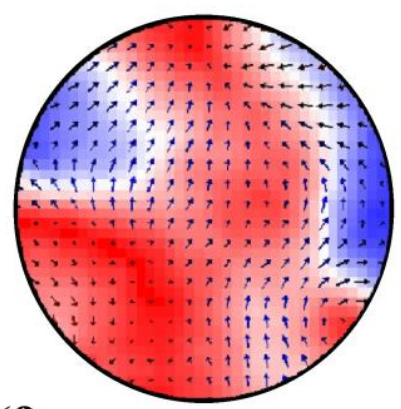

(f)

Fig. 4. Spatial magnetization configurations before the current pulse is switched off. The arrows refer to the $y$-component of the magnetization (blue positive and red negative), whereas the background colors refer to the $z$ component (red positive direction and blue negative direction). The letter "V" indicates vortexes, while "AV" refers to antivortexes. (a) Pulse time of 3 ns. (b) Pulse time of 2.8 ns. (c) Pulse time of 2.6 ns. (d) Pulse time of 3.4 ns. (e) Spatial magnetization configuration after the current pulse is removed for a pulse time (e) $t=3 \mathrm{~ns}$ and (f) $t=2.6 \mathrm{~ns}$.
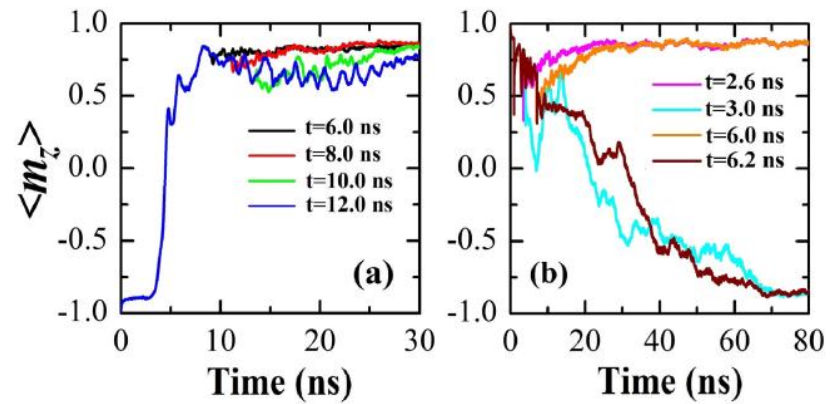

Fig. 5. Time domain plot for the $z$-component of the magnetization for different time pulses at $T=300 \mathrm{~K}$. (a) $\mathrm{P} \rightarrow \mathrm{AP}$ switching process. (b) $\mathrm{AP} \rightarrow \mathrm{P}$ switching process. 